\title{
Simultaneous estimation of plasma parameters from spectroscopic data of neutral helium using least square fitting of CR-model
}

\author{
Jalaj Jain · Ram Prakash · Gheesa Lal Vyas · Udit Narayan Pal • \\ Malay Bikas Chowdhuri • Ranjana Manchanda • Nilanjan Halder • \\ Yaduvendra Choyal
}

Received: 6 February 2014/ Accepted: 3 November 2014/Published online: 10 December 2014

(C) The Author(s) 2014. This article is published with open access at Springerlink.com

\begin{abstract}
In the present work an effort has been made to estimate the plasma parameters simultaneously like-electron density, electron temperature, ground state atom density, ground state ion density and metastable state density from the observed visible spectra of penning plasma discharge (PPD) source using least square fitting. The analysis is performed for the prominently observed neutral helium lines. The atomic data and analysis structure (ADAS) database is used to provide the required collisional-radiative (CR) photon emissivity coefficients (PECs) values under the optical thin plasma condition in the analysis. With this condition the estimated plasma temperature from the PPD is found rather high. It is seen that the inclusion of opacity in the observed spectral lines through PECs and addition of diffusion of neutrals and metastable state species in the CRmodel code analysis improves the electron temperature estimation in the simultaneous measurement.
\end{abstract}

\section{J. Jain}

Universidad de Talca, Talca, Chile

J. Jain

Comisión Chilena de Energía Nuclear, Santiago, Chile

R. Prakash · U. N. Pal

Microwave Tubes Division, CSIR-Central Electronics and

Engineering Research Institute, Pilani 333031, India

G. L. Vyas $(\bowtie) \cdot$ N. Halder

Manipal University Jaipur, Jaipur 303007, India

e-mail: glvyas27@gmail.com

M. B. Chowdhuri - R. Manchanda

Institute for Plasma Research, Bhat, Gandhinagar 382428, India

Y. Choyal

School of Physics, Devi Ahilya Vishwavidyalaya,

Indore 452017, India
Keywords Penning discharge $\cdot$ Collisional-radiative model $\cdot$ Least square fitting $\cdot$ Opacity

\section{Introduction}

Penning plasma discharge (PPD) devices have been studied significantly during last few decades. This device was originally proposed by Penning as a low-pressure manometer (i.e., cold cathode or penning gauge) [1]. This has later been adopted to function as an ion source [2-4]. Penning ion sources have been used for variety of applications, such as sputtering and evaporation of surfaces, electromagnetic separation of isotopes and fusion applications $[5,6]$. The PPD devices are also attractive due to their extensive use as charged particles trapping [7, 8], light emitting sources [9], etc. In the applications of light emitting sources it has recently been proposed that the PPD device can be used to calibrate a VUV spectrometerdetector system [10]. In fact, the PPD device can generate spectral radiations in the visible and VUV region simultaneously and visible radiations analysis can help in VUV intensity calibration once the desired basic plasma parameters are obtained from the visible spectra.

In a penning discharge, the plasma is produced between two end cathodes and a shell anode, with power derived from DC high voltage power supply. The electron confinement is maintained very high in this geometry by end cathodes (electrostatic confinement) and a pair of permanent magnets, which produces an axial magnetic field (magnetic confinement). The high electron energies in the source gas results in highly ionized gas and secondary electron release from the cathode. The source produces a large number of bright emission lines even at low pressures and the spectral distribution depends on the combination of 
discharge gas, applied magnetic field, applied power, cathode material, etc. [11-13]. Although penning discharge sources have been used for many applications and different sources have been researched, there exist no direct diagnostic which can provide the basic plasma parameters measurement simultaneously like-electron density, electron temperature, ground state atom density, ground state ion density, metastable state density, etc.

In the case of magnetic field environment, and small geometries of the penning discharge devices where sheathsheath intersection could be prominent, the metallic probe diagnostics can lead erroneous results and are not much useful [14-16]. The optical emission spectroscopy (OES) [17-19], on the other hand, is an advanced technique which plays an important role to complement the metallic probe diagnostic techniques because this is a passive diagnostic and does not perturb the plasma environment locally. Nevertheless, the interpretation of the spectra of OES is not straight-forward. The delicacy lies in the complex nature of the plasma and subsequently the manifold atomic processes. In fact, it requires an estimation of the population density of the specific excited energy state wherefrom specific wavelength radiation is emitted. This is possible by solving a set of coupled rate equitation's by including all the atomic processes at a particular condition.

Based on the assumptions and requirements there exist three models which are quite popular in the plasma physics community for the plasma parameter estimations, namely, Corona, local thermodynamic equilibrium (LTE) and collisional-radiative (CR) models [20, 21]. The corresponding Corona and LTE models are best used for low $\left(<10^{10} \mathrm{~cm}^{-3}\right)$ and high $\left(10^{15} \mathrm{~cm}^{-3}>\right)$ electron density regimes of the plasmas. The CR-model is a general model which works in the intermediate density regime and it is also applicable in the Corona and the LTE model regimes self-consistently even if the density is of the order of above-mentioned low and high density limits [21]. This makes it more useful in the most spectroscopic studies.

The CR-model based line-ratio technique is often used to estimate the electron plasma density and temperature $[22,23]$. The line ratio method is used by the earlier workers to estimate the electron plasma density and temperature in the PPD source, which are typically $\sim 10^{11} \mathrm{~cm}^{-3}$ and $\sim 2-5 \mathrm{eV}$ [24], respectively. However, the line-ratio method is constrained by the fact that identified line-ratios must be sensitive either to electron density or to electron temperature for estimation of one plasma parameter at a time. Also, we have to identify suitable spectral line pairs to be used, which should follow either ionizing or recombining plasma conditions [25]. In this work, an effort has been made to develop a spectroscopic method which works on least square fitting of the absolute intensities of the observed visible neutral helium spectral lines from a PPD source and the CR-model-based estimated intensities using atomic data and analysis structure (ADAS) code [26] to find out the basic plasma parameters simultaneously from a large number of visible spectral lines. This method is applicable for all the spectral lines observed at a time.

The analysis is performed with thin plasma condition and electron density, electron temperature, ground state atom density, ground state ion density and $2{ }^{3} \mathrm{~S}$ metastable state density are the parameters estimated. The estimated plasma temperature from the PPD is found high in the simultaneous measurement at which the neutral helium may not even survive and seems to be unrealistic. The analysis is improved by the inclusion of opacity in the observed spectral lines through photon emissivity coefficients (PECs) and also addition of diffusion of neutrals and metastable state species in the CR-model code. This helps in adequate electron temperature estimation in the simultaneous measurement.

\section{Experimental setup}

The PPD source (VUV Source SD-01 from Jobin-Yvon, France) consists of two cathodes with an anode ring between them. The two cathodes face each other and the emitted electrons that oscillate between the cathodes fall through the central ring. Because of the field of the permanent magnets located behind the cathodes the charged particles are confined within the discharge volume. Electrons that leave the cathode are confined by the magnetic field. The experimental arrangement of $1 \mathrm{~cm}$ cylindrical plasma column length is shown in the Fig. 1.

Helium gas discharge is produced in the penning plasma source. The gas pressure is controlled by the needle valve and discharge current is controlled with the control knob in the PPD source power supply. The PPD source is cooled during operation by flowing demineralised water at $1 \mathrm{lit} /$ min. During the experiment the working pressure is kept $\sim 4 \times 10^{-4}-1 \times 10^{-2} \mathrm{mbar}$ and the discharge current $\sim 20-150 \mathrm{~mA}$. The spectroscopic data are recorded in the visible region using visible spectrometer at various filled pressures for fixed discharge current and also at different discharge currents and at fixed fill pressure. The analysis of the spectra which is recorded at different discharge currents 20, 64, 80, 90 and $100 \mathrm{~mA}$ and at constant filled pressure $4 \times 10^{-3}$ mbar is discussed.

The light emitted during the discharge is transmitted by an optical fiber and fed to the visible monochromator (wavelength resolution $\sim 2.5 \AA$ ) fitted with a charge coupled device (CCD) camera-based acquisition system to record the visible emission lines. The recorded helium lines are He I $7281.3 \AA\left(2 \mathrm{p}^{1} \mathrm{P}^{0}-3 \mathrm{~s}^{1} \mathrm{~S}\right)$, He I $7065.1 \AA$ 
Fig. 1 Schematic diagram of the penning discharge source

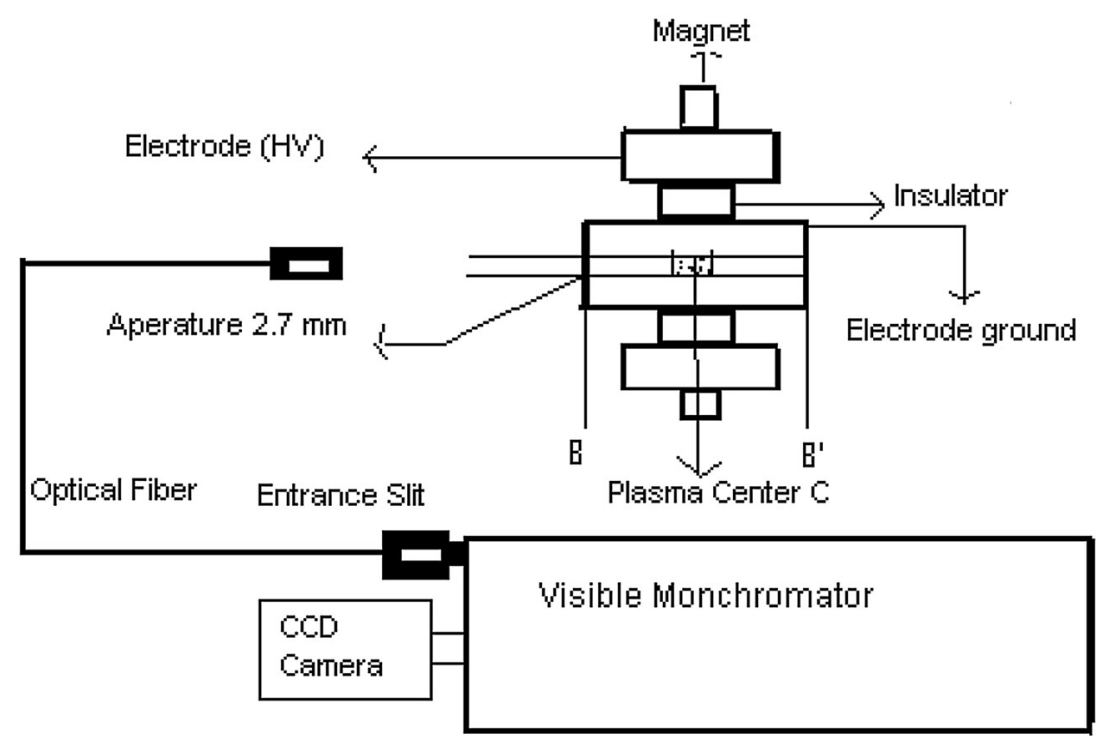

$\left(2 \mathrm{p}^{3} \mathrm{P}^{0}-3 \mathrm{~s}^{3} \mathrm{~S}\right)$, He I $6678.1 \AA\left(2 \mathrm{p}^{1} \mathrm{P}^{0}-3 \mathrm{~d}^{1} \mathrm{D}^{0}\right)$, He I $5875.6 \AA$ $\left(2 \mathrm{p}^{3} \mathrm{P}^{0}-3 \mathrm{~d}^{3} \mathrm{D}\right)$, He I $5047.7 \AA\left(2 \mathrm{p}^{1} \mathrm{P}^{0}-4 \mathrm{~s}^{1} \mathrm{~S}\right)$, He I $5015.7 \AA$ $\left(2 \mathrm{~s}^{1} \mathrm{~S}-3 \mathrm{p}^{1} \mathrm{P}^{0}\right)$, He I $4921.9 \AA\left(2 \mathrm{p}^{1} \mathrm{P}^{0}-4 \mathrm{~d}^{1} \mathrm{D}\right)$, He I $4713.1 \AA$ $\left(2 \mathrm{p}^{3} \mathrm{P}^{0}-4 \mathrm{~s}^{3} \mathrm{~S}\right)$, He I $3964.7 \AA\left(2 \mathrm{~s}^{1} \mathrm{~S}-4 \mathrm{p}^{1} \mathrm{P}^{0}\right)$, and He I $3888.6 \AA$ $\left(2 \mathrm{~s}^{3} \mathrm{~S}-3 \mathrm{p}^{3} \mathrm{P}^{0}\right)$. Since He I $5015.7 \AA\left(2 \mathrm{~s}^{1} \mathrm{~S}-3 \mathrm{p}^{1} \mathrm{P}^{0}\right)$ line is affected by resonance scattering [27], we have worked with 9 lines out of these 10 observed lines. In fact, this effect has not been considered in the CR-model calculations of the ADAS code [26]. Using remaining nine lines a simple procedure using least square fitting is developed to determine the plasma parameters.

\section{Model analysis}

With an assumption of average electron density and temperature in $1 \mathrm{~cm}$ plasma column length, the photon intensity of a spectral line for such an averaged measurement can be written from the CR-model as [22, 28],

$$
\begin{aligned}
\tilde{I}\left(\lambda_{\text {ul }}\right)= & \left\{\tilde{N}_{e} \tilde{N}_{i} P E \tilde{C}_{\text {reco }}+\tilde{N}_{e} \tilde{N}_{g} P E \tilde{C}_{\text {exc }}+\tilde{N}_{e} \tilde{N}_{m 1} P E \tilde{C}_{\text {meta }, 1}\right. \\
& \left.+\tilde{N}_{e} \tilde{N}_{m 2} P E \tilde{C}_{\text {meta }, 2}\right\} .
\end{aligned}
$$

Here $\mathrm{PE} \tilde{C}_{\text {reco }}, \operatorname{PE} \tilde{C}_{\text {exc }}, \operatorname{PE} \tilde{C}_{\text {meta, } 1}$ and $\mathrm{PE} \tilde{C}_{\text {meta }, 2}$ are the average values of PECs, having units $\mathrm{cm}^{3} / \mathrm{s}$ for recombination, excitation, singlet metastable state $\left(2{ }^{1} \mathrm{~S}\right)$ and triplet metastable state $\left(2{ }^{3} \mathrm{~S}\right)$, respectively. The values $\tilde{N}_{e}, \tilde{N}_{i}, \tilde{N}_{g}$, $\tilde{N}_{m 1}$ and $\tilde{N}_{m 2}$ represent the average densities of electron, ground state ion, ground state atom, singlet and triplet metastable states of helium atoms, respectively. Moreover, PECs are the complicated functions of the electron density and temperature and can be obtained from the ADAS database for a given value of electron density and temperature. Since the life time of singlet metastable state is of the order of characteristic time scale of change of plasma,
ADAS does not consider $2{ }^{1} \mathrm{~S}$ level as a metastable state and hence Eq. (1) can be written as,

$\tilde{I}\left(\lambda_{\mathrm{ul}}\right)=\left\{\tilde{N}_{e} \tilde{N}_{i} P E \tilde{C}_{\text {reco }}+\tilde{N}_{e} \tilde{N}_{g} P E \tilde{C}_{\mathrm{exc}}+\tilde{N}_{e} \tilde{N}_{m 2} P E \tilde{C}_{\text {meta }, 2}\right\}$.

Assuming quasi-neutral and purely ionizing condition for plasma, a merit function $S$ is defined to use the least square fitting technique for all nine observed spectral lines as,

$S=\sum_{j=1}^{9}\left[I_{\exp _{j}}-\left(\tilde{N}_{e} \tilde{N}_{g} P E \tilde{C}_{\text {excj }}+\tilde{N}_{e} \tilde{N}_{m 2} P E \tilde{C}_{\text {meta }, 2 \mathrm{j}}\right)\right]^{2}$,

where, $I_{\exp _{j}}$ represents experimentally observed intensities and the terms in the small bracket represent the theoretical intensities derived from the CR-model. In Eq. 3 there are two fitting parameters $\tilde{N}_{g}$ and $\tilde{N}_{m 2}$ for a given set of electron density and temperature. The most suitable values of these fitting parameters can be obtained by minimizing the merit function $S$ with respect to the parameters $\tilde{N}_{g}$ and $\tilde{N}_{m 2}$, i.e.,

$$
\begin{aligned}
& \frac{\partial S}{\partial N_{g}}=0 \text { and } \frac{\partial S}{\partial N_{m 2}}=0 \Rightarrow \tilde{N}_{g}=\frac{I_{2}-(C / B) I_{1}}{B-A C / B} \\
& \text { and } \tilde{N}_{m 2}=\frac{I_{1}-\tilde{N}_{g} A}{B} \text {, } \\
& A=\sum_{j}\left(\tilde{N}_{e} \mathrm{PE} \tilde{C}_{\text {excj }}\right)^{2}, B=\sum_{j} \tilde{N}_{e} \mathrm{PE} \tilde{C}_{\text {excj }} \times \tilde{N}_{e} \mathrm{PE} \tilde{C}_{\text {meta }, 2_{j}}, \\
& C=\sum_{j}\left(\tilde{N}_{e} \mathrm{PE} \tilde{C}_{\text {meta }, 2_{j}}\right)^{2}, \\
& I_{1}=\sum_{j} \tilde{N}_{e} \mathrm{PE} \tilde{C}_{\operatorname{excj}} I_{\exp j}, I_{2}=\sum_{j} \tilde{N}_{e} \mathrm{PE} \tilde{C}_{{\text {meta }, 2_{j}}} I_{\exp _{j}} .
\end{aligned}
$$




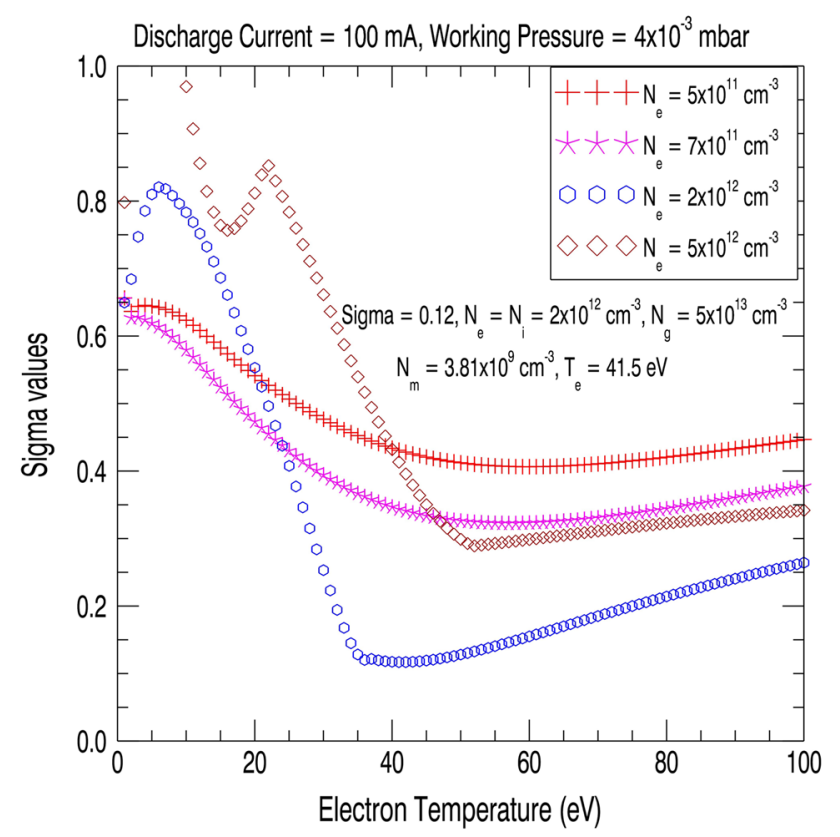

Fig. 2 Mismatch parameter $\sigma$ for the discharge current $100 \mathrm{~mA}$ at constant pressure of $4 \times 10^{-3}$ mbar under thin plasma condition

From Eqs. (4) and (5) the values of $\tilde{N}_{g}$ and $\tilde{N}_{m 2}$ are estimated. Theoretical intensities $\tilde{I}\left(\lambda_{\mathrm{ul}}\right)$ are calculated for all the nine lines, which are termed as $\tilde{I}_{\text {calj }}$, using the obtained values of $\tilde{N}_{g}$ and $\tilde{N}_{m 2}$ for larger grid points of $\tilde{N}_{e}$ and $\tilde{T}_{e}$. To predict the best fit basic plasma parameters, these calculated intensities $\tilde{I}_{\text {calj }}$ are compared with the experimental values $\left(I_{\text {expj }}\right)$ for all the nine lines $(j=1,9)$ through a mismatch parameter,

$\sigma=\sqrt{\frac{1}{9} \sum_{j=1}^{9}\left(\frac{I_{\exp j}-I_{\mathrm{cal} j}}{I_{\exp j}}\right)^{2}}$,

The minimum in the mismatch parameter $(\sigma)$ gives the best-fit values of $\tilde{N}_{e}, \tilde{T}_{e}, \tilde{N}_{i}, \tilde{N}_{g}$ and $\tilde{N}_{m 2}$.

\section{Results and discussion}

The mismatch parameters obtained at fixed working pressure $4 \times 10^{-3}$ mbar and different discharge currents 20 , 64, 80, 90 and $100 \mathrm{~mA}$ are $0.35,0.33,0.14,0.15$ and 0.12 , respectively. Figure 2 shows the plot of mismatch parameter at different temperatures and densities for fixed discharge current $100 \mathrm{~mA}$ and working pressure $4 \times 10^{-3}$ mbar. For this operating plasma condition, the predicted basic plasma parameters are $\tilde{N}_{g}=5 \times 10^{13} \mathrm{~cm}^{-3}$, $\tilde{N}_{m, 2}=3.8 \times 10^{9} \mathrm{~cm}^{-3}, \quad N_{e} \approx \tilde{N}_{i}=2 \times 10^{12} \mathrm{~cm}^{-3}$, $T_{e}=41.5 \mathrm{eV}$ with a minimum $\sigma \sim 0.12$. At this condition a comparison between estimated intensities and

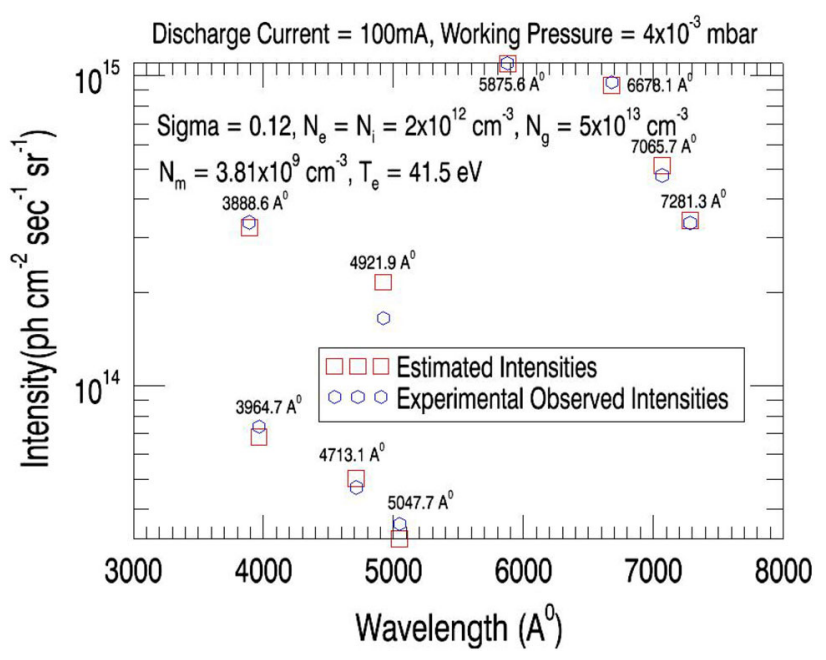

Fig. 3 Comparison of theoretical and experimental intensities at $100 \mathrm{~mA}$ and $4 \times 10^{-3}$ mbar of pressure under thin plasma condition

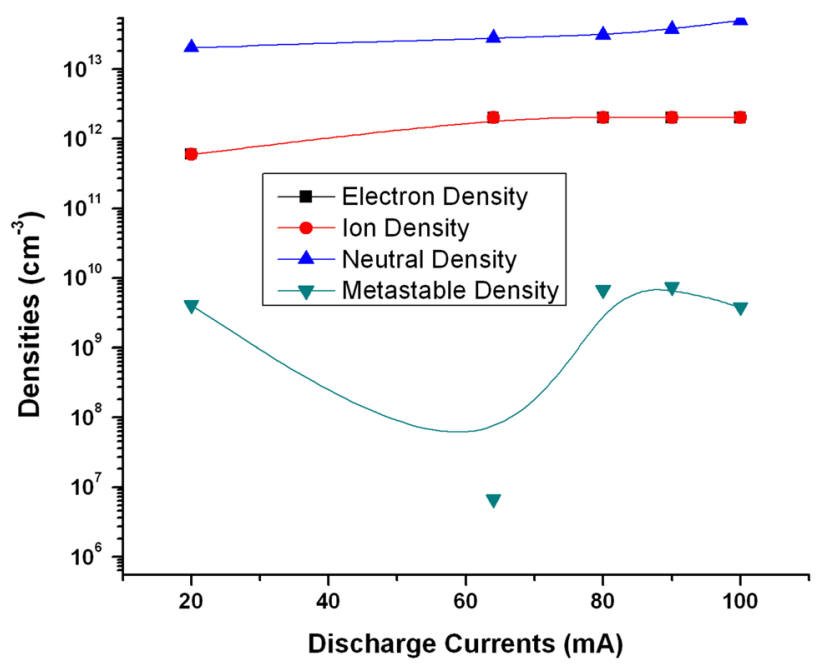

Fig. 4 The variation of neutral helium, triplet metastable state density, ion and electron densities at fixed pressure $4 \times 10^{-3}$ mbar and with different discharge currents under thin plasma condition

experimentally observed intensities is also shown in Fig. 3. Though the fit with intensities is good but the estimated electron temperature is quite high. At such temperature neutral helium may not survive that shows a discrepancy in temperature estimation.

To further elaborate these results the variation of the metastable state density and ground state atom density for different discharge currents and fixed pressure is shown in Fig. 4. It is observed that the metastable state density decreases up to $64 \mathrm{~mA}$ discharge current and then increases. This behavior of metastable state does not seem obvious. Indeed, at fixed pressure, an increment in the discharge current suggest the consumption of more neutrals in creation of larger electrons to draw the higher discharge 
currents, which may enhance the effective recombination process over the excitation/ionization process. Moreover, the transitions happening due to recombination process may lead in production of larger metastable populations. It is also seen from the figure that the neutral density increases with discharge current. This seems ambiguous because for larger discharge current the neutrals have to be ionized more and hence the neutral density should decrease. Therefore, this analysis needs one or more considerations to predict the correct behavior of the plasma discharge.

It is to be emphasized that the above calculations are carried out assuming optically thin and diffusion less plasma conditions which may not necessarily be correct for all the applications. In fact, most spectroscopic diagnostic techniques require optically thin plasma for model simplifications, which is very near homogeneous along the line-of-sight and remains in steady-state for the duration of the observation. However, if the plasma is optically thick for the radiation coming from the plasma, the re-absorption (opacity) of the photons may cause the wrong information about the estimated plasma parameters [29, 30]. So, the aforesaid discrepancies in temperature and neutral density obtained in the results may be due to the effect of the opacity and also the diffusion of neutral and metastable states. The ADAS code calculates opacity affected PECs on the basis of escape factor methodology [26, 29-33]. Basically, inclusion of escape factors causes a net reduction in Einstein's A coefficients, i.e., it modifies the atomic transition probability values. The effect of self-absorption not only changes the net emergent flux but also changes the population of excited states and hence the effective rate coefficients.

The changes in excited state population and ionization balance due to re-absorption of radiation are then derived using the ADAS 208 code [26]. According to the used experimental setup the following parameters are used to calculate the opacity affected photo emissivity coefficients in the ADAS code: density distribution (parabolic), plasma geometry (cylindrical), line profile (Doppler), neutral temperature $(600 \mathrm{~K})$, plasma length $(1 \mathrm{~cm})$ and neutral density $\left(5 \times 10^{13} \mathrm{~cm}^{-3}\right)$. The detailed description of the model used for opacity calculations is given in the ADAS user manual [26]. The opacity effect has been considered for the following transitions and spectral lines: He I 7281.3 $\AA\left(2 \mathrm{p}^{1} \mathrm{P}^{0}-3 \mathrm{~s}^{1} \mathrm{~S}\right)$, He I 7065.1 $\AA\left(2 \mathrm{p}^{3} \mathrm{P}^{0}-3 \mathrm{~s}^{3} \mathrm{~S}\right)$, He I 6678.1 $\AA\left(2 \mathrm{p}^{1} \mathrm{P}^{0}-3 \mathrm{~d}^{1} \mathrm{D}^{0}\right)$, He I $5875.6 \AA\left(2 \mathrm{p}^{3} \mathrm{P}^{0}-3 \mathrm{~d}^{3} \mathrm{D}\right)$, He I $5047.7 \AA\left(2 \mathrm{p}^{1} \mathrm{P}^{0}-4 \mathrm{~s}^{1} \mathrm{~S}\right)$, He I $5015.7 \AA\left(2 \mathrm{~s}^{1} \mathrm{~S}-3 \mathrm{p}^{1} \mathrm{P}^{0}\right)$, He I $4921.9 \AA\left(2 \mathrm{p}^{1} \mathrm{P}^{0}-4 \mathrm{~d}^{1} \mathrm{D}\right)$, He I $4713.1 \AA\left(2 \mathrm{p}^{3} \mathrm{P}^{0}-4 \mathrm{~s}^{3} \mathrm{~S}\right)$, He I $3964.7 \AA\left(2 \mathrm{~s}^{1} \mathrm{~S}-4 \mathrm{p}^{1} \mathrm{P}^{0}\right)$, and He I 3888.6 $\AA$ ( $\left.2 \mathrm{~s}^{3} \mathrm{~S}-3 \mathrm{p}^{3} \mathrm{P}^{0}\right)$.

A diffusion time scale $\sim 10^{-4} \mathrm{~s}$ is also taken into account in the ADAS code to introduce the diffusion of neutral and metastable states to the wall. The diffusion time scale was estimated for our geometry using radius of the cylinder $(a=0.5 \mathrm{~cm})$, zero order Bessel's function $J_{0}$ and the diffusion coefficient $D$ for helium discussed earlier [34]. To illustrate it clearly, we have assumed purely diffusive transport in cylindrical symmetry. In fact, if the impurity species' are no longer fed by external source in the plasma, their density decays and the radial impurity profile first rearrange their shape and eventually decrease self-similarly with a time (i.e., diffusion time scale) given by the following expression [34],

$\tau_{0}=\frac{a^{2}}{J_{0}^{2}(0) D}$

In this expression, the particle diffusion coefficients are still not very well-known from theory because they are often determined by instabilities in plasma (i.e., anomalous nature in general) and are usually described from the experiments. One basically requires diagnostic line excited data and atomic coefficients for total radiation to compare final results with bolometer kind of diagnostics. All such calculations are quite complicated and are done through various plasma transport codes, e.g., STRAHL [35, 36].

In spectroscopy, the diffusion overall effects the population of the excited species, which is directly related with the ground state and metastable populations and indirectly related with the purely or partially ionized plasmas [37]. In our case, we have added diffusion for partially ionized plasma and only for metastable state, which can affect the populations of the particular energy level (i.e., excited state) during the measurements. This may be the region for wrong estimation of plasma parameter during model understanding without opacity and diffusion.

After this, the theoretical intensities and accordingly the mismatch parameters $(\sigma)$ with the experimental values are recalculated by repeating the same procedure as explained above while including the effect of opacity in the PEC's and diffusion of neutrals and metastable states to the wall. The obtained minimum mismatch parameters $(\sigma)$ at working pressure $4 \times 10^{-3}$ mbar and different discharge currents 20,64, 80, 90 and $100 \mathrm{~mA}$ are 0.62, 0.66, 0.65, 0.65 and 0.69 , respectively. The variation of the $\sigma$ with electron temperatures and different electron densities is shown in Fig. 5. At this condition the estimated values of $\tilde{N}_{g}$ and $\tilde{N}_{m, 2}$ are $4.22 \times 10^{13}$ and $1.02 \times 10^{13} \mathrm{~cm}^{-3}$, respectively, while the calculated values of electron temperature, electron density and ion density are $T_{e}=2.0 \mathrm{eV}$, $N_{e} \approx \tilde{N}_{i}=7 \times 10^{10} \mathrm{~cm}^{-3}$, respectively.

A comparison between experimental and estimated intensities is shown in Fig. 6. From this figure it can be seen that the miss-match between the two spectral lines 6678.1 and $7281.3 \AA$ has increased in the simultaneous measurement but it improves the temperature estimation. 


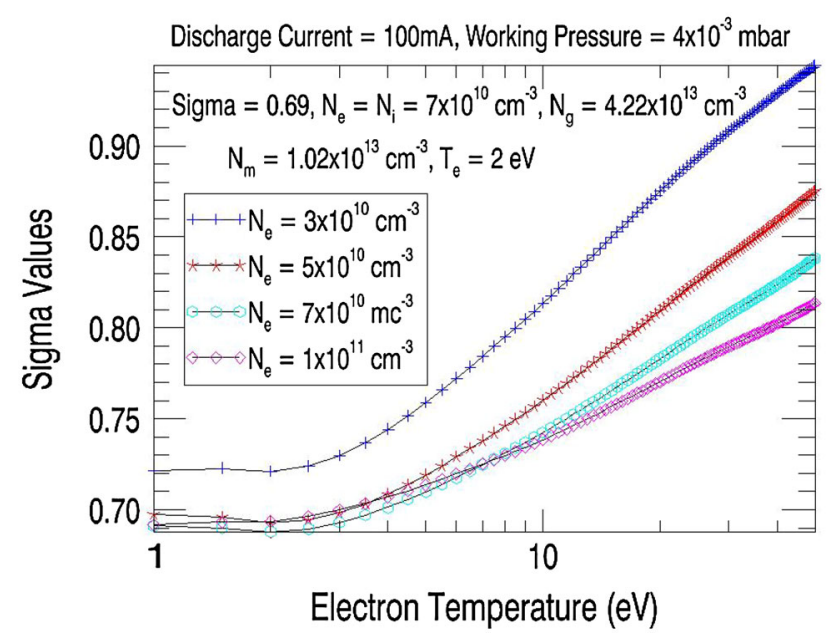

Fig. 5 Mismatch parameter $\sigma$ for the discharge current $100 \mathrm{~mA}$ at constant pressure of $4 \times 10^{-3}$ mbar with opacity and diffusion effects considerations

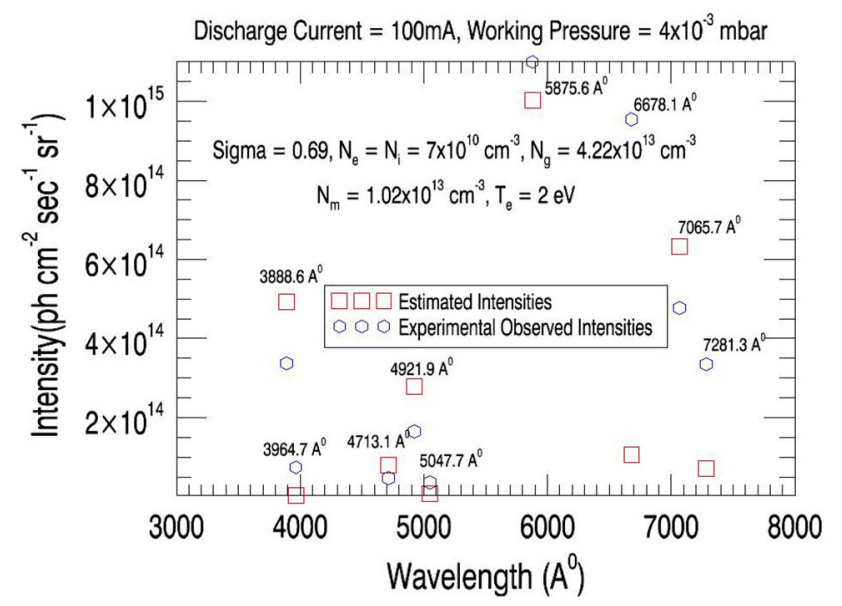

Fig. 6 Comparison of theoretical and experimental intensities at $100 \mathrm{~mA}$ discharge current and $4 \times 10^{-3}$ mbar working pressure under opacity and diffusion considerations

More efforts are needed to understand the exact behavior of 6678.1 and $7281.3 \AA$ spectral lines in penning type of plasma discharges. The non-Maxwellian electron consideration in the CR-model may provide an exact solution, however, such state-of-art researches are underway [38].

Based on the improved calculations the variation of metastable and neutral densities with the discharge currents at fixed working pressure is shown in the Fig. 7. It is seen that with the increasing discharge current the neutral density decreases and the metastable state density increases. This is the predictable behavior of neutrals and metastable states because the enhancement in the plasma discharge current requires more ionization which may increase the recombination processes and hence there might be an increment in metastable state densities and also decrement in the neutral density.

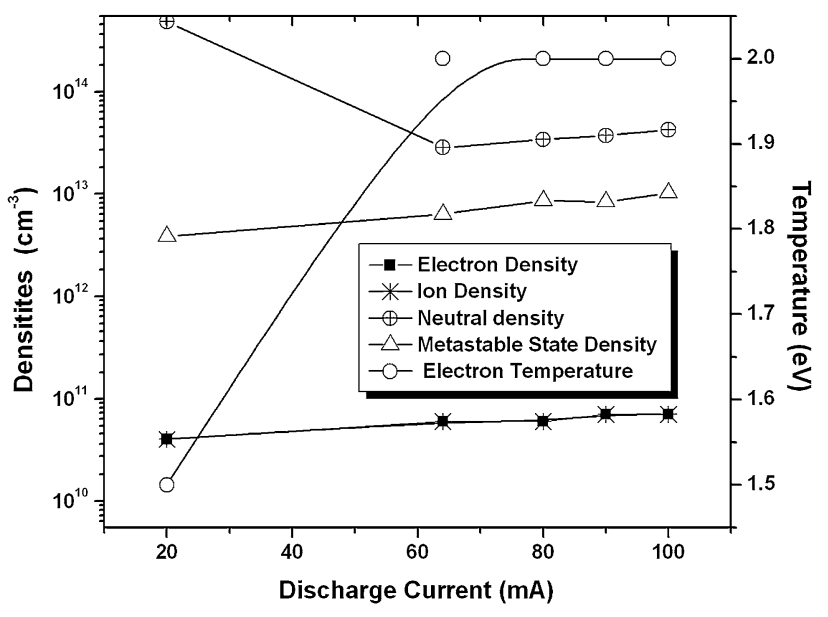

Fig. 7 The variation of neutral helium, triplet metastable state density, ion and electron densities and electron temperature at fixed pressure $4 \times 10^{-3}$ mbar and with different discharge currents including opacity and diffusion effects

\section{Conclusions}

In this study, a procedure to calculate the basic plasma parameters like $-N_{e}, T_{e}, \tilde{N}_{i}, \tilde{N}_{g}$ and $\tilde{N}_{m}$ simultaneously from a penning discharge source using least square fitting of spectroscopic data and ADAS code is presented. In case of the optically thin and diffusion less plasma assumption, the obtained electron temperature is rather high. Hence the assumption of optically thin and diffusion less plasma is not applicable. The opacity calculations and diffusion of metastable and neutral are then added in the method using ADAS code to estimate the plasma parameters correctly. With this condition, the neutral density decreases and metastable population of helium $2{ }^{3} \mathrm{~S}$ state increases with discharge currents, which is quite consistent.

Acknowledgments The authors thankfully acknowledge the Spectroscopy Diagnostics Division of Institute for Plasma Research, Bhat, Gandhinagar, India for providing the experimental facility and data. Thanks are also due the ADAS consortium, Prof. H. P. Summers, Prof. Kurt Behringer and Prof. Martin O' Mullane for helping us to use and learn the ADAS database. Authors also thankfully acknowledge Board of Research in Fusion Science and Technology (BRFST) for the financial support for this work under projects NFP/ DIAG/2 and NFP/DAQ/A09/02.

Open Access This article is distributed under the terms of the Creative Commons Attribution License which permits any use, distribution, and reproduction in any medium, provided the original author(s) and the source are credited.

\section{References}

1. Penning, F.M.: Ein neues manometer für niedrige gasdrucke, insbesondere zwischen $10^{-3}$ und $10^{-5} \mathrm{~mm}$. Physica. (Amsterdam) 4, 71 (1937) 
2. Rikoski, R.A.: Compact penning discharge ion source for plasma diffusion studies. J. Phys. E: Sci. Instrum. 5, 540 (1972)

3. Roth, J.R.: Industrial Plasma Engineering, IOP publishing, London, pp. 204 (1995)

4. Gow, J.D., Foster, J.S.: A high-intensity pulsed ion source. Rev. Sci. Instrum. 24, 606 (1953)

5. Hillis, D.L., Morgan, P.D., Groth, J.K.M., Stamp, M.F., Hellermann, M., Ehrenberg, Von., Kumar, V.: Tritium concentration measurements in the Joint European torus by optical spectroscopy of a penning gauge. Rev. Sci. Instrum. 70, 359 (1999)

6. Hillis, D.L., Klepper, C.C., Hellermann, M., Ehrenberg, Von J., Finken, K.H., Mank, G.: Deuterium-tritium concentration measurements in the divertor of a tokamak via a modified Penning gauge. Fusion Eng. Des. 347, 34-35 (1997)

7. Brown, L.S., Gabrielse, G.: Geonium theory: physics of a single electron or ion in a penning trap. Rev. Mod. Phys. 58, 233 (1986)

8. Major, F.G., Gheorghe, V.N., Werth, G.: Charged Particle Traps: Physics and Techniques of Charged Particle Field Confinement. Springer, Berlin (2005)

9. Heise, C., Hollandt, J., Kling, R., Kock, M., Kuehne, M.: Radiometric characterization of a penning discharge in the vacuum ultraviolet. Appl. Opt. 33, 5111 (1994)

10. Prakash, R., Jain, J., Kumar, V., Manchanda, R., Agarwal, B., Banerjee, S., Chowdhuri, M.B., Vasu, P.: Calibration of a VUV spectrograph by collisional-radiative modelling of a discharge plasma. J. Phys. B At. Mol. Opt. Phys. 43, 144012 (2010)

11. Hirsch, E.H.: On the mechanism of the Penning discharge. Br. J. Appl. Phys. 15, 1535 (1964)

12. Feng, P.X., Weiner, B.: High abundance of metastable helium atoms for diagnostic applications. J. Phys. B At. Mol. Opt. Phys. 37, 3265 (2004)

13. Finley, S.D., Bowyer, S., Paresce, F., Malina, R.F.: Continuous discharge penning source with emission lines between 50 a and 300 a. Appl. Opt. 18, 649 (1979)

14. Hutchinson, I.H.: Principle of Plasma Diagnostics, Cambridge Univ. Press, Cambridge, p. 60 (2002)

15. Godyak, V., Demidov, V.I.: Probe measurements of electronenergy distributions in plasmas: what can we measure and how can we achieve reliable results? J. Phys. D Appl. Phys. 44, 233001 (2011)

16. Chen, F.F.: Langmuir Probe Diagnostics, Mini-Course on Plasma Diagnostics, IEEE-ICOPS meeting, Jeju (2003)

17. Griem, H.R.: Principles of Plasma Spectroscopy, Cambridge Univ Press, Cambridge (1997)

18. Kunze, H.J.: Introduction to Plasma Spectroscopy. Springer, Heidelberg (2009)

19. Fujimoto, T.: Plasma Spectroscopy. Clarendon Press, Oxford (2004)

20. Bates, D.R., Kingston, A.E., McWhirter, R.W.P.: Recombination between electrons and atomic ions. I. optically thin plasmas. Proc. R. Soc. Lond. A 267, 297 (1962)
21. Fantz, U.: Basics of plasma spectroscopy. Plasma Sources Sci. Technol. 15, S137 (2006)

22. Prakash, R., Vasu, P., Kumar, V., Manchanda, R., Chowdhuri, M.B., Goto, M., Aditya Team: Characterization of helium discharge cleaning plasmas in ADITYA tokamak using collisionalradiative model code. J. Appl. Phys. 97, 43301 (2005)

23. Goto, M.: Collisional-radiative model for neutral helium in plasma revisited. J. Quant. Spectrosc. Radiat. Transfer 76, 331 (2003)

24. Feng, P.X., Andruczyk, D., James, B., Takiyama, W.K., Namba, S., Oda, T.: High-density metastable helium atoms produced by Penning-type discharges. Plasma Source Sci. Technol. 12142 (2003)

25. Sasaki, S., Goto, M., Kato T., Takamura, S.: Line Intensity Ratios of Helium Atom in an Ionizing Plasma. NIFS-DATA-49 (2008)

26. Summers, H.P.: Atomic data and analysis structure user's manual. JET-IR 06, JET Joint undertaking, Abingdon (1994)

27. Sasaki, S., et al.: Helium I Line Intensity Ratios in a Plasma for the Diagnostics of Fusion Edge Plasmas. NIFS-DATA-346 (1995)

28. Prakash, R., Pütterich, T., Dux, R., Kallenbach, A., Fischer, R., Behringer, K., ASDEX Upgrade Team.: Spectroscopic Divertor Characterization and Impurity Influx Measurements in ASDEX Upgrade Tokamak, vol. 10/31, IPP (2006)

29. Behringer, K.: Escape Factors for Line Emission and Population Calculations, vol. 10/11, Report IPP, (1998)

30. Behringer, K., Fantz, U.: The influence of opacity on hydrogen excited-state population and applications to low-temperature plasmas. New J. Phys. 2, 23 (2000)

31. Irons, F.E.: The escape factor in plasma spectroscopy-I. The escape factor defined and evaluated. JQSRT 22, 1 (1979)

32. Irons, F.: E, The escape factor in plasma spectroscopy-II. The case of radiative decay JQSRT 22, 21 (1979)

33. Boivin, R.F., Kline, J.L., Scime, E.E.: Electron temperature measurement by a helium line intensity ratio method in helicon plasmas. Phys. Plasmas 8, 5303 (2001). (and references therein)

34. Behringer, K.: Spectroscopy of fusion plasmas: methods and atomic data requirements. In: Ram Prakash. (ed) IAEA International Workshop on Challenges in Plasma Spectroscopy for Future Fusion Research Machines, Jaipur, India (2008) vol. 1, pp. 3. Excel India Publisher, Delhi (2008)

35. Behringer, K.: Description of the Impurity Transport Code "STRAHL", Report JET-R(87) 08, (1987)

36. Dux, R.: STRAHL User Manual, vol. 10/30, Report IPP (2006)

37. Prakash, R.: Plasma and the Role of Atomic Physics in its Spectroscopic Diagnostic. In: Srivastava, R., Choubisa, R. (eds.) Atomic and Molecular Physics: Introduction to Advanced Topics, pp. 203-235. Narosa Publishing House, New Delhi (2012)

38. Bingham, R., Kellett, B.J., Bryans, P., Summers, H.P., Torney, M., Shapiro, V.D., Spicer, D.S., O’Brien, M.: A new mechanism explaining the asymmetric x-ray emission from Supernova Remnant PKS 1209-52. Astrophys. J. 601, 896 (2004) 